\title{
Utilization of Insecticide Treated Bed Nets among Mothers Attending MCH/FP in Webuye District Hospital, Bungoma County, Kenya
}

\author{
Taratisio Ndwiga, Robert M. Kei, Odiver W. Dancan \\ Department of Environmental Health, School of Public Health, Moi University, Eldoret, Kenya \\ Email: taratisiondwiga@yahoo.com
}

Received 16 April 2014; revised 25 May 2014; accepted 12 June 2014

Copyright (C) 2014 by authors and Scientific Research Publishing Inc. This work is licensed under the Creative Commons Attribution International License (CC BY). http://creativecommons.org/licenses/by/4.0/

(c) (i) Open Access

\section{Abstract}

Malaria is the leading cause of morbidity and mortality in Kenya, with close to 70 percent (24 million) of the population at risk of infection. It affects people of all age groups: children under five years of age and pregnant women living in malaria endemic regions who are vulnerable. The main objective was to assess the utilization of the insecticide treated bed nets among the mothers attending MCH/FP in Webuye District Hospital, Bungoma County, Kenya. This research was based at the Webuye District Hospital, Bungoma County, Kenya from February to May, 2013. Sample size included 40 adult mothers attending MCH/FP aged 18 years and above during the study period. The design of the study was cross-sectional where sampling technique employed was non-probabilistic, purposive sampling. Data was collected by interviews using structured questionnaire which was administered by the researchers. SPSS version 16 was employed in Data analysis. The association between the overall knowledge about ITN use and malaria attack and level of education was tested and correlation between knowledge about malaria and ITNs utilization was calculated. Nearly all mothers attending MCH/FP had knowledge about ITNs nets and used it, with majority, $82.5 \%$ of the respondents used it for protection and $75 \%$ knew the importance of ITNs which were for malaria prevention. A majority of mothers attending MCH/FP were aware of ITNs and used it. Malaria morbidity was influenced by various factors including frequency of ITN use and most respondents interviewed had contracted malaria once before. The difference was found to be highly statistically significant between the overall knowledge about ITN use and malaria attack and level of education $\left(\chi^{2}=58.7, p=0.000\right)$. There was a significantly moderate positive correlation between total knowledge and ITN utilization $(r=0.449 \& p=0.000)$. The same was for the frequency of use but it was found to be in a weak magnitude, $(r=0.223 \& p=0.000)$. There was a strong positive correlation between knowledge about risk which is exposed to the case of non-utilization and the overall knowledge $(r=0.853 \& p=0.000)$. Based on the above results, it's recommended that the Ministry 
of Health increase knowledge of effective malaria prevention and treatment methods in communities where misconceptions and use of unproven prevention and treatment methods are common.

\title{
Keywords
}

\author{
Malaria, Insect Treated Nets (ITNs), Mother Child Health and Family Planning (MCH \& FP), Indoor \\ Residual Spraying, Integrated Vector Management
}

\section{Introduction}

Malaria is the leading cause of morbidity in Kenya, with close to 70 percent (24 million) of the population at risk of infection [1]. Although malaria affects people of all age groups, children under five years of age and pregnant women living in malaria endemic regions are most vulnerable.

The human toll that malaria exacts and the economic and social impacts are devastating: sick children miss school, working days are lost, and tourism suffers. Malaria becomes a self-perpetuating problem, where the disease prevents growth of the human and economic capital necessary to bring the disease under control.

The Kenya vision 2030 goal for health sector provide equitable, affordable, quality health services to all Kenyans. The goal also aims to restructure the health care delivery system to shift the emphasis from curative to preventive health care. Malaria prevention and control activities in Kenya are guided by the National Malaria Strategy (NMS) and the National Health Sector Strategic Plan [2]. Strategy aims to achieve national and international malaria control targets.

Interventions adopted in Kenya include Vector control-using insecticide treated nets (ITNs) and indoor residual spraying (IRS), implementation of these strategies was expected to reduce the level of malaria infection and consequent death in Kenya by 30\% by the year 2006 and sustain the improved level of control until 2010. Untreated nets and window screening have long been considered using protection methods against mosquito and other insects. The use of insecticide-treated nets (ITNs) has been shown to be effective methods of reducing sever malaria and when used by all or most members of the community may reduce malaria transmission.

Scaling up the use of ITNs and protecting $80 \%$ of children under five and pregnant women against malaria in Africa by the year 2010 is one of the targets set at the Abuja summit by African Heads of State in 2000. Therefore to scale up the use of ITNs, mechanisms of delivering ITNs to vulnerable groups have been used, in Kenya (routine clinic delivery, mass campaigns retail social marketing, and the commercial sector) used.

By the end of 2008, an estimated 16 million nets had been documented as distributed in Kenya through multiple channels since 2002, targeting children under five and pregnant women.

The government recognizes the children $<5$ years of age as a risk group and recommends that this group be protected by sleeping under the ITNs. In 2008, the national target for the proportion of children under five sleeping under ITN was $80 \%$. The level of education plays a major role in use of mosquito nets by women of the reproductive age. $48 \%$ of pregnant women with no education used mosquito nets the previous night compared to $53 \%$ of those with secondary education according to [3].

Based on series of field studies of the effect of ITNs on malaria morbidity and mortality in sub-Saharan Africa, promotion of use of ITNs has emerged as a key intervention for malaria control. Every year, malaria kills 1 2 million people and infects 300 - 500 million. 90\% of deaths occur in sub-Saharan Africa. The disease is present in over 100 countries, threatening $40 \%$ of the world's population.

The Kenya Malaria Indicator Survey [4] revealed that in endemic areas, $74 \%$ of households own at least one net while $39 \%$ own more than one. In the same areas $58 \%$ of all households own at least one ITN and $28 \%$ own more than one ITN. For households in epidemic-prone areas, 59\% own at least one net; while $33 \%$ own more than one. In the same areas $48 \%$ own at least one ITN and $24 \%$ own more than one ITN.

The data show that $51 \%$ of children $<5$ years of age slept under any net the night preceding the survey, but only $39 \%$ slept under an ITN [5]. About $61 \%$ of children under five in urban areas used mosquito net the night preceding the survey, compared with $50 \%$ in rural areas. Endemic areas have the highest use of mosquito nets (58\%) and ITNs (46\%). Areas in which two mass net distribution campaigns were conducted show a higher ITN usage among children under five than non-campaigns ones. That is, in areas where there was no campaign, only 
$27 \%$ of children under five had slept under an ITN the night preceding the survey whereas $44 \%$ of those residing in campaign areas had done so [5].

The survey shows that pregnant women were more likely than all women within the reproductive age to have slept under a mosquito net: About $51 \%$ of pregnant women slept under a net the night preceding the survey compared with $45 \%$ of all women of reproductive age. Similarly, $40 \%$ of pregnant women had slept under an ITN compared with $32 \%$ of all women of reproductive age. One woman in two (50\%) in urban areas slept under a net the night preceding the survey; compared with two in five rural women (43\%).

Also, $42 \%$ of pregnant women in urban areas slept under an ITN compared with $39 \%$ of their rural counterparts [5]. Malaria prevalence in older children (26\% of four-year olds) is lower than that in younger children (32\% of children below one year). Prevalence is highest in malaria endemic areas (40\%) and (25\%) lowest in epidemic and highland areas [5].

A large proportion of Kenyans continue to carry one of the highest preventable burdens of ill health in the world. Much of this burden can be lifted and prevented with existing knowledge and resources within the country. Malaria remains the first cause or death for children under five in Africa-it kills one child every thirty seconds worldwide. Malaria is the leading cause of morbidity and mortality in Kenya, with close to 70\% (24 million) of the population at risk of infection (1). The human toll that malaria exacts and the economic and social impact are devastating: sick children miss school, working days are lost, and tourism suffers.

Area is stable malaria zone having altitudes ranging from 0 to 13,000 meters around Lake Victoria in Western Kenya. Children under the age of five and pregnant mothers are at high risk of contracting malaria due to reduced immunity, therefore they most need protection from mosquito bites to reduce the morbidity rate. The current ITN ownership of 0.8 per household in Kenya is far from universal access defined as 2 nets per household.

Despite having well defined national policies and reform agenda whose overriding strategies are focused on improving health care delivery services through efficient and effective health management systems and reform, there has not been a breakthrough in improving the situation of usage and management of the ITNs among the society members vulnerable to malaria disease as well entrapped in the vicious cycle of poverty and ill health.

The situation is further complicated by the continuous existence of the malaria disease which the health sector and other related organization entrusted with the task of facing out the disease. This uses numerous of government revenue yearly which could be significant in developing or investing in food production including agriculture and manufacturing industries. The community systems are faced with the challenge of coping with the growing demand for care, in the face of deepening poverty and dwindling resources [1].

Malaria not only cuts lives short but has a huge socio-economic impact: patients are often bed ridden and incapable of carrying out normal daily activities. The disease causes considerable loss of income and places a heavy burden on families, health systems and society as a whole.

The government of Kenya spends a lot of money on curative medical services. These costs are ever on the increase hence the renewed focus on primary health care activities through programs such as the community strategy which is part of the NHSSP II of the KEPH program. To ensure efficiency and minimization of wastage of funds, it is prudent to assess the utilization of the ITNs among mothers and whether they truly embrace it.

Yearly, malaria kills 1 - 2 million people and infects 300 - 500 million. 90\% of deaths occur in sub-Saharan Africa. The disease is present in over 100 countries, threatening $40 \%$ of the world's population. Based on series of field studies of the effect of ITNs on malaria morbidity and mortality in sub-Saharan Africa, promotion of use of ITNs has emerged as a key intervention for malaria control. ITNs coverage at Webuye Health records stands at $79 \%$ and research shows utilization of ITNs is most important for malaria eradication.

Even if the government funding of health projects is indispensable, it is necessary to out monitoring and interim evaluation of these projects to ensure that funds are not being wasted and are being directed to the actual activities they were meant to accomplish by searching the community. The viability of the projects has be assessed as early as possible, especially if the projects are being rolled out for the first time e.g. on trial or pilot status and then later getting rolled out nationwide.

The ITN supply is done in every hospital especially to mothers attending the MCH who are supposed to have treated nets to avoid mosquito bite, this is a project started by the ministry of health. This necessitates interim monitoring to determine the extent/degree to which the objectives have been accomplish as far as prevention of malaria among mothers is concerned and to establish any constrains that may be dragging the process behind. This includes determining the knowledge attitudes and perceptions of mothers towards usage of insecticides treated nets. 
The findings of this research may be used as a tool in formulating policies and legislations by the government to control the malaria cases that is a continuous problem in major hospitals including Webuye District Hospital by maintaining education on effective utilization of ITNs and the importance.

Same research findings may also be used by the disease surveillance department when seeking funds from the government, and NGO's to ensure that malaria is made a thing of the past by ensuring the same percentage of mothers without ITNs get access to it to reduce the disease burden on the reproductive age mothers.

The main objective was to assess the utilization of the insecticide treated nets among mothers attending $\mathrm{MCH} / \mathrm{FP}$ in Webuye District Hospital, with specific objectives as to assess the use of ITNs by mothers attending $\mathrm{MCH} / \mathrm{FP}$, to determine the malaria morbidity amongst mothers with ITNs and to determine the malaria morbidity amongst mothers without ITNs.

Surveys of pregnant women in eastern India showed that bed nets were widely available and used, although other prevention measures, such as chemoprophylaxis and ITNs, were underused [6].

Creative strategies that combine distribution of ITNs with other key services such as immunization days or antenatal visits should be explored. Where resources are limited, distribution at a subsidized price should be considered, an approach successfully tried elsewhere in India [7].

Insecticide-treated nets and intermittent preventive treatment (IPT) with sulfadoxine-pyrimethamine are recommended for control of malaria during pregnancy in endemic areas in Africa, 45 (96\%) of 47 countries surveyed had a policy for distribution of ITNs for pregnant women; estimated coverage in 2007 being 4.7 million (17\%) of 27.7 million pregnancies at risk of malaria in 32 countries. 39 (83\%) of 47 countries surveyed had an intermittent preventive treatment policy; in 2007, an estimated 6.4 million (25\%) of 25.6 million pregnant women received at least one dose of treatment and 19.8 million (77\%) visited an antenatal clinic (31 countries). Estimated coverage being lowest in areas of high-intensity transmission of malaria [8].

In 2007, there were about 32 million pregnancies in malaria-endemic areas in sub-Saharan Africa [9]. WHO's recommendation for malaria prevention and control during pregnancy in areas of stable malaria transmission in Africa is a package of IPT and ITNs with effective management of clinical malaria and anemia, which is commonly delivered through collaboration between malaria and reproductive-health programme [10].

The Roll Back Malaria initiative aims to ensure that all pregnant women receive intermittent preventive treatment and at least $80 \%$ of people at risk from malaria use ITNs in areas of high-intensity transmission by 2010, including those who are pregnant [11]. Specific targeting of pregnant women aimed at increased use of ITNs began in sub-Saharan Africa earlier than did IPT, but coverage is much lower than targets set by the RBM initiative [12].

The RBM initiative guidelines published in 2004 and revised in 2009 recommend "proportion of pregnant women who slept under an ITN the previous night" as the indication of ITN use [11]. The definition of ITN coverage being ITN used during the previous night as reported by women, which has several limitations. Reported use might not be actual use, or use can be seasonal depending on the perceived nuisance of mosquitoes, and thus the time of survey can affect the rates obtained [13]. Use of both ITNs and IPT during pregnancy seems to be of benefit. Treated nets have the additional benefit of providing protection to the mother before, during, and after pregnancy, and potentially to infants [14].

Some countries aim to protect vulnerable population with ITNs and IRS. Spraying can potentially affect the coverage of ITNs; for example, in an area where spraying is done, people might be less motivated to use treated nets. In the future, when indoor residual spraying might be increasingly used, it will be important that this indicator is standardized and documented at national and sub national levels, and separately reported for vulnerable groups, such as children younger than 5 years or pregnant women [15].

Integrated vector management (IVM) also provides a framework for improved personal protection/prevention strategies that combine the use of environmental management tools/physical barriers with chemical tools for new synergies, e.g. (ITNs). Trials using insecticides-treated bed-nets in some malaria-endemic African countries have shown very substantial reduction in child and infant mortality [16].

A recent US audit of malaria prevention in Benin summarizes the shortfalls of a commodities-driven approach: USAID had delivered 705,000 nets-worth $\$ 4.1$ to 800 health Centers across the country sitting in storage for nearly eight months. Such top-down approaches are fast being replaced by bottom-up, community-driven initiatives. Over the last two decades, morbidity and mortality from malaria have increased. In response to this growing challenge, international development agencies, spearheaded by the WHO, founded the Roll Back Malaria (RBM) initiative, a global partnership for prevention and control of malaria. 
Over 1.2 million people-overwhelmingly children-died of malaria in 2002. This figure reflects an increase in mortality in absolute terms over the previous year, according to data published by the World Health Organization (WHO) in its World health report 2004 (4). Also in 2002, the total burden of disease from malaria was estimated by WHO to be 46,486,000 Disability Adjusted Life Years (DALYs) lost. This figure represents the combined toll of death, illness, and disability from the disease.

Over $85 \%$ of malaria deaths, disease, and disability occur in the African Region, with the south-East Asia and Eastern Mediterranean Regions being the second and third hardest-hit by malaria. Some experts suggest an even higher incidence of malaria cases than that reported by countries, both in Africa and globally. Malaria is endemic in India and constitutes a major public health challenge. An estimated 2 - 3 million malaria cases resulting in 500 - 800 deaths are reported annually nationwide, with 95\% of India's population at risk of infection, as has been demonstrated in Sub-Saharan Africa and Thailand [17].

Based on series of field studies of the effect of ITNs on malaria morbidity and mortality in sub-Saharan Africa, promotion of use of ITNs emerged as key intervention for malaria control. Malaria is caused by four species of plasmodium protozoa (single-cell parasites): plasmodium falciparum, vivax, ovale and malariae. Of the four species, plasmodium falciparum is responsible for most deaths. The parasites transmission by Anopheline mosquitoes by climate and geography, and is often highest during the rainy season.

Symptoms of malaria include fever, shivering, pain in the joints, headaches, repeated vomiting, convulsions and coma. If left untreated, the disease-particularly that caused by $P$. falciparum may cause progress to serve malaria and sometimes death.

In the cot d'Ivoire, farmers diagnosed as sick from malaria for more than two days out of a growing season had $47 \%$ lower yields and 53\% lower revenues than farmers who missed no more than two days of work (8 - 10). In general, families highly affected by disease of various kinds may turn from growing higher value crops to less labour demanding and yield-sensitive products, with consequences for household income and nutrition (10). An analysis of economic growth over 25 year found that countries with intense malaria had rates of GDP growth that were $1.3 \%$ lower than those in comparable countries with less intense malaria. Another analysis found that countries with more than $50 \%$ of the population living at risk of malaria [16].

Chloroquine, developed in 1934, is very cheap, easy to administer and has few side-effects. For a while, it was the ideal anti-malaria, but its effectiveness has decreased dramatically in the last few decades: resistance to Chloroquine now reaches over $90 \%$ in many parts of Africa. Resistance to other first-line drugs is also growing rapidly-for example, resistance to sulphadoxine-pyrimethamine is already higher than $60 \%$ in several regions of Burundi and Uganda.

Anti-malarial drugs are often administered on their own (mono-therapy), but the World Health Organization now strongly recommends switching protocols to include an artemisinin-based combination therapy (ACT). By hitting different biochemical targets of parasites, drug combination is more affective, allow for shorter treatment courses, and protect each individual drug from resistance. It is now widely agreed that the best current treatment solutions is ACT. Artemisinin derivatives, which are extracted from a Chinese plant-are fast-acting, highly potent and complimentary to other classes of treatment. To date, no resistance to ACT has been reported.

There are several types of nets available. Nets may vary by size, material, and/or treatment. Most nets are made of polyester but nets are also available in cotton, polyethylene, or polypropylene. Currently, only pyrethroids insecticides are approved for use on ITNs. These insecticides have very low mammalian toxicity but highly toxic to insect and have a rapid knock-down effect, even at very low doses. Pyrethroids have a high residual effect: they do not rapidly break down unless washed or exposed to sunlight.

To maintain the efficacy of ITNs, the nets must be treated at intervals of 6 - 12 months, more frequently if the nets are washed. Retreatment is done by simply dipping nets in a mixture of water and insecticides and allowing the nets to dry in a shady place. Kits for retreating nets are available in most countries.

The need for frequent retreatment is one of the most difficult barriers to full implementation of ITNs use in endemic countries. The additional cost of the insecticides and the lack of understanding of its importance result in very low retreatment rates in most African Countries. Several companies have developed long-lasting ITNs that theoretically retain lethal concentration of insecticide for the life of the net (3 - 5 years). CDC is currently testing several of these products in Kenya.

\section{Methodology}

The study was conducted in Webuye district Hospital Bungoma county, Western province, Kenya, where the 
Study Design of a cross-sectional study was used. The study population was all the mothers living in the catchment area of Webuye District Hospital and the Target Population being Mothers attending MCH/FP aged 18 years and above during study period. The sample size of 40 mothers was interviewed.

Purposive Sampling was employed and only those subjects found in the study area during the study period were interviewed. Adults aged 18 years and above in the study area who attended MCH/FP during the study period; Mother before, during and after pregnancy, and potentially to infants. Adults as described above but who were unable to provide informal consent, unable to provide reliable data were not interviewed.

Data was collected by interviews by use of structured questionnaire which was administered by the researcher. Data Analysis was done for the frequencies and percentages using SPSS and data presented in form pie charts, graphs, and tables. The association between the overall knowledge about ITN use and malaria attack and level of education was tested using the $\chi^{2}$ and the Correlation between knowledge about malaria and ITNs utilization was test using the correlation co-efficient.

Permission was sought from the hospital administration and Consent sought from the key informants after being assured of confidentiality. The respondents were recruited primarily among women attending MCH/FP at Webuye District Hospital and thus failed to capture the views of women in comparatively marginalized, remote tribal populations.

\section{Results}

The respondents were 18 - 41 years of age with a few (7.5\%) greater than 36 years of age (Figure 1), and most $42.5 \%$ between 18 - 23 years. On the of education, 5\% had no schooling; another $42.5 \%$ had completed primary school with $37 \%$ attending secondary school, and $15 \%$ with post-secondary education (Figure 2). On occupation Most respondents (32.5\%) were housewife engaged in housework; 15\% were involved in agriculture, 12.5\% others worked in saloon and other unskilled labor jobs, or commercial activities and $7.5 \%$ were teachers. For the religion, Most of the respondent 80\% were Protestants with 17.5\% catholic and 2.5\% Muslim.

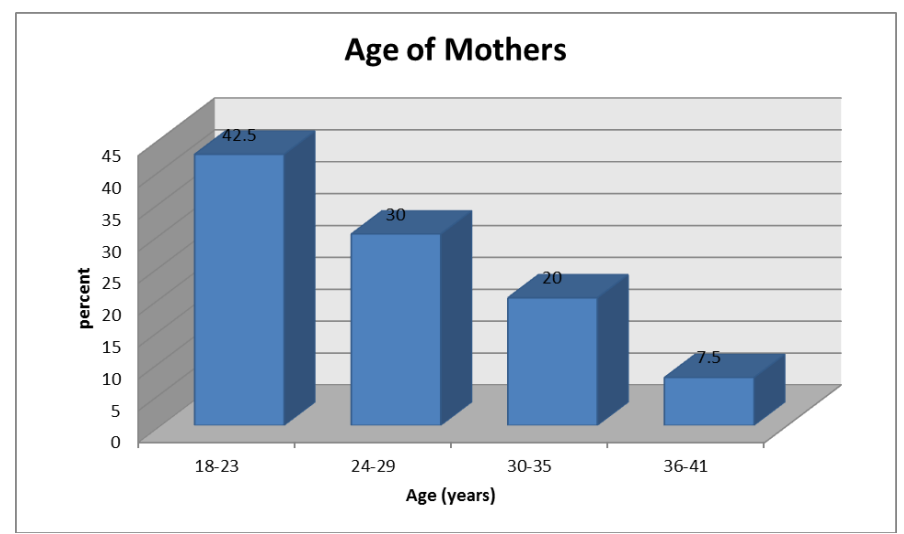

Figure 1. Age of mothers.

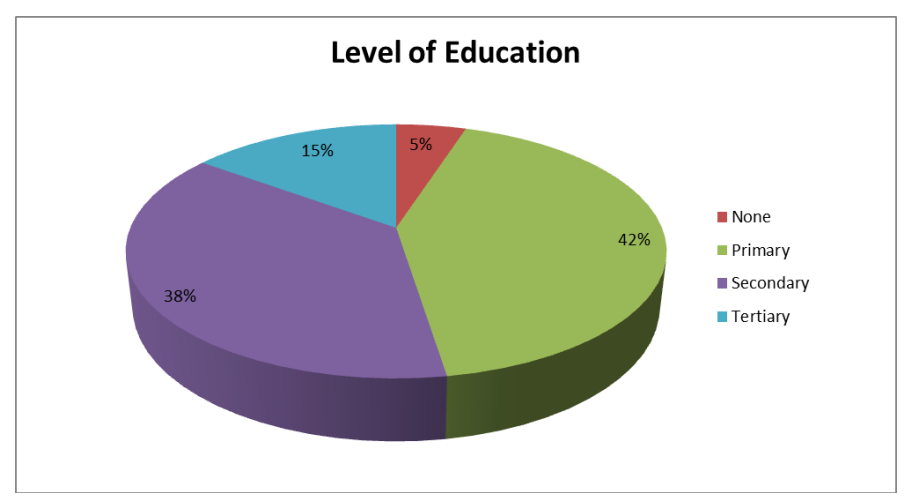

Figure 2. Level of education. 
On the Knowledge on ITNs (Figure 3), all respondents had an idea about the ITNs during the time of the study (Figure 4), saying they have heard about ITNs. For the ITN Ownership, for personal protection, most respondents $90 \%$ possessed while few $10 \%$ said that they did not have ITN. On the use of ITNs, $82.5 \%$ respondents and most respondents used the ITNs for covering themselves and the child, with few 5\% admitting they use ITNs for restricting chicken and $2.5 \%$ for sieving water from river which was incorrect. On the time of use of ITNs, Most respondents 50\% said they used ITNs at night, 27\% used day and night with few 10\% using sometimes which is seasonal and $2.5 \%$ protected themselves and child when they remember.

For the Importance of ITNs (Figure 5), a number of respondents 75\% demonstrated ITNs importance saying they used to prevent mosquito bites, $17 \%$ mentioned they prevented insect nuisance, and a few respondents $7.5 \%$ said it was a requirement for mother and child advocated by the government.

On the Knowledge on Malaria (Figure 6), all respondents 100\% demonstrated full knowledge of malaria, and linked malaria transmission to a mosquito bite. For the Causes of Malaria, Most respondents $72.5 \%$ linked malaria transmission with a mosquito bite and $10.5 \%$ mentioned it was caused by anopheles mosquito, with $15 \%$ saying cold weather being the cause. $2.5 \%$ mentioned much sugar cane, which is an incorrect cause of malaria transmission. On previous Malaria attacks, $52.5 \%$ admitted they had been attacked by malaria in the past one year during the time study was conducted while almost half $47.5 \%$ said they had not experienced malaria attack for the past one year.

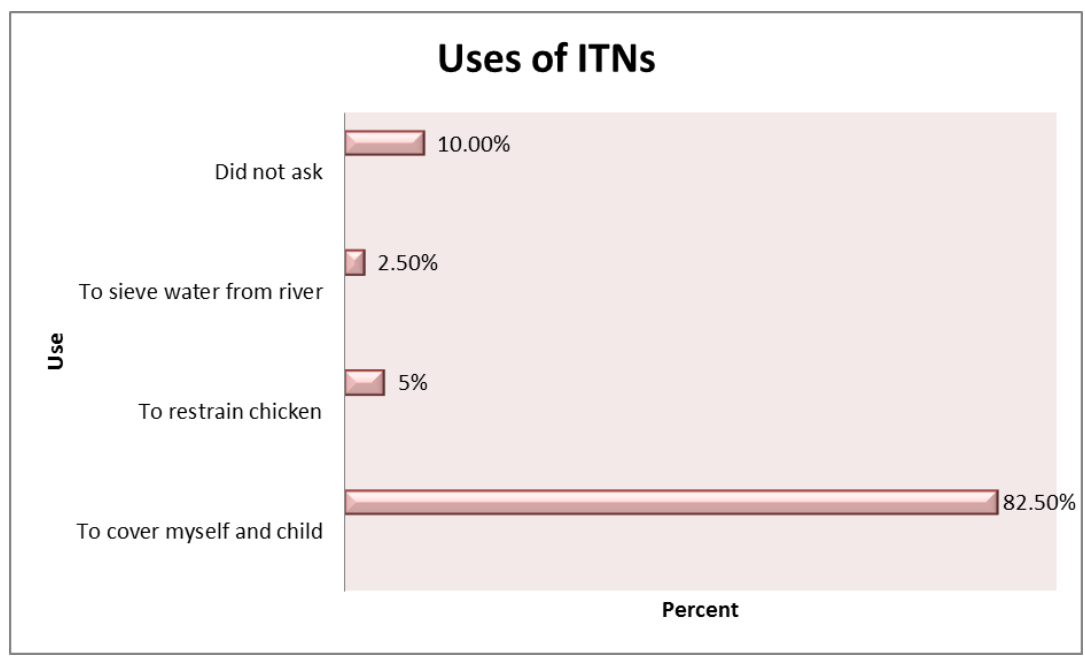

Figure 3. Uses of ITNs.

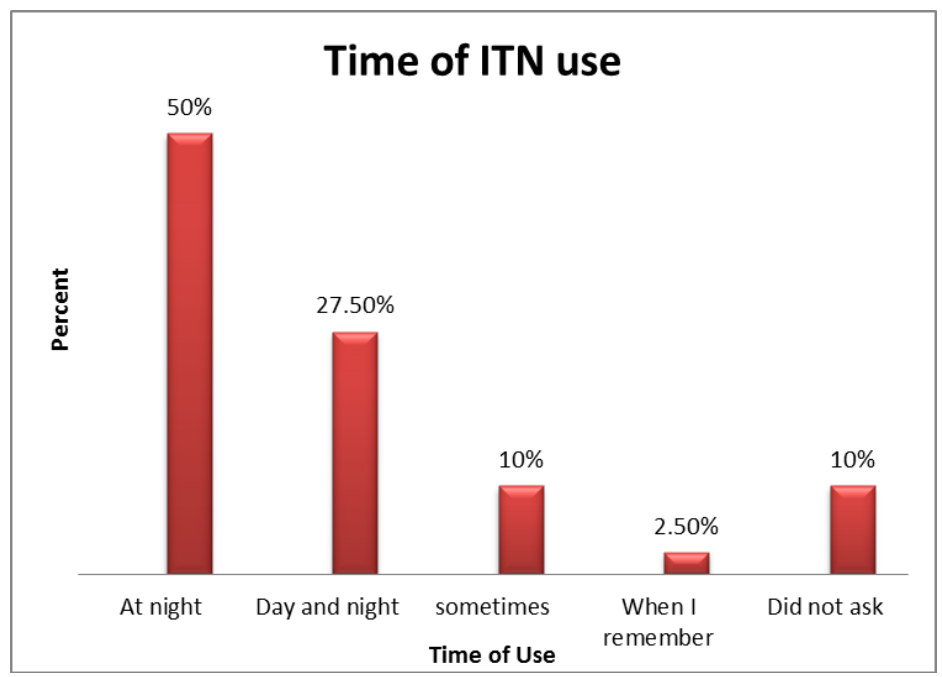

Figure 4. Time of ITN use. 


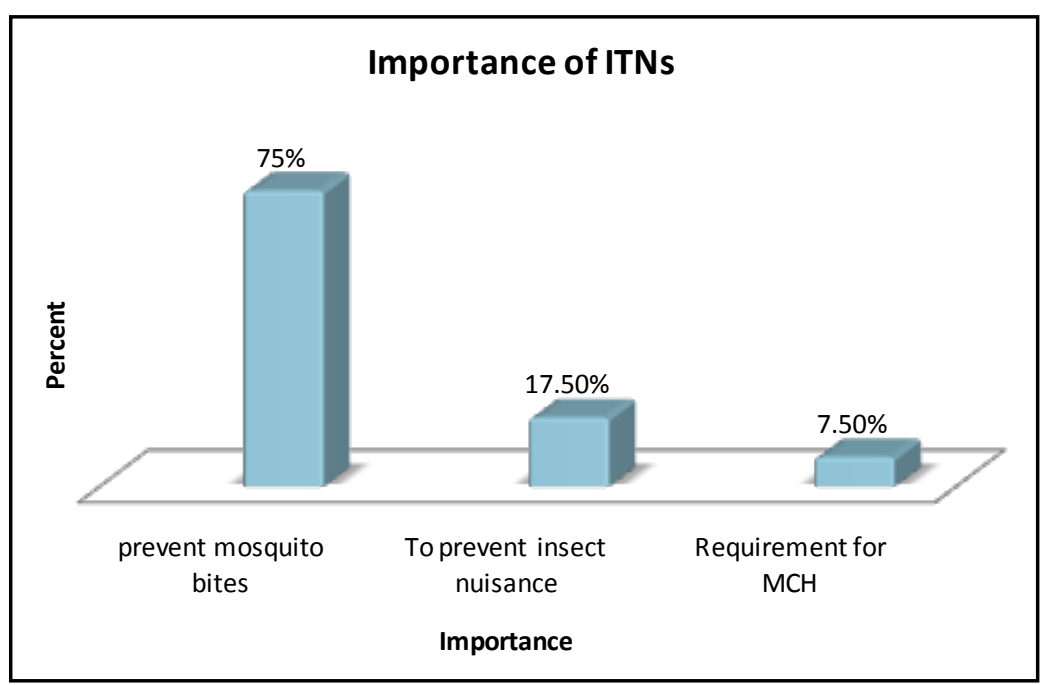

Figure 5. Importance of ITNs.

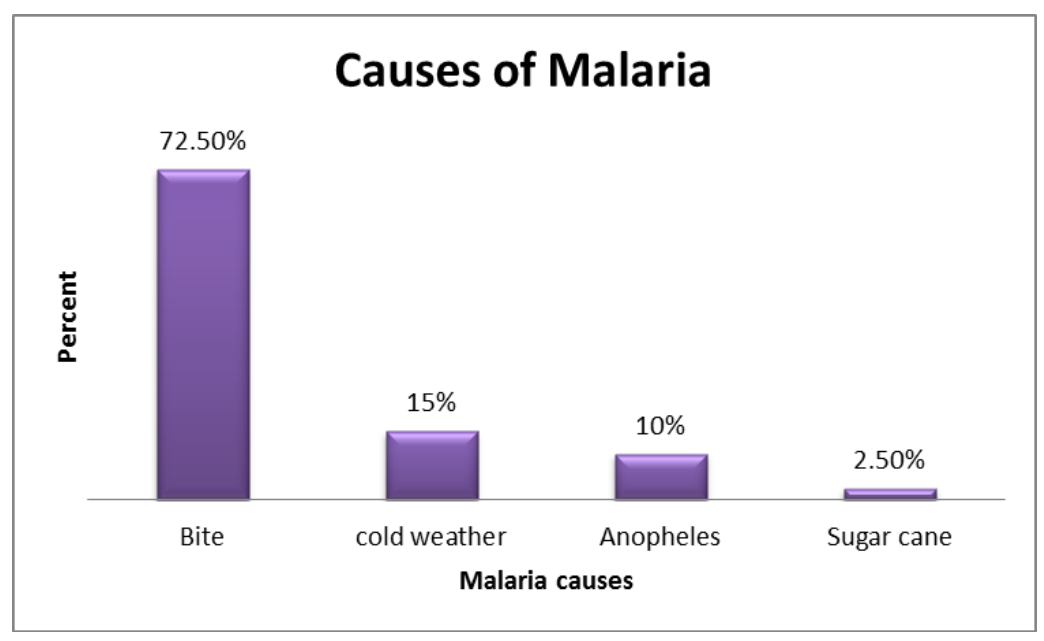

Figure 6. Causes of malaria.

On the Frequency of Malaria attacks (Figure 7), of the 52.5\% respondents attacked (20\% of them attacked only once, and $12.5 \%$ attacked more than thrice). On whether they received treatment or never sought treatment (Figure 8), among those who received malaria attacked $47.5 \%$ received treatment while few $5 \%$ never sought treatment.

Regarding the overall knowledge about ITN use and malarial attack, all respondents had an idea about the ITNs during the time of the study, saying they have heard about ITNs. For the ITN Ownership, for personal protection, most respondents $90 \%$ possessed while few $10 \%$ said that they did not have ITN. $15 \%$ of tertiary level sample had a good level of knowledge compared to $42 \%$ of the primary level sample compared to $5 \%$ who never attended any school and 38\% who attended up to secondary level of education. The difference was found to be highly statistically significant between the overall knowledge about ITN use and malaria attack and level of education $\left(\chi^{2}=58.7, \mathrm{p}=0.000\right)$.

\section{Correlation between Knowledge about Malaria and ITNs Utilization}

There was a significant moderate positive correlation between total knowledge and ITN utilization $(r=0.449$ \& $\mathrm{p}=0.000)$. The same was for the frequency of use but it was found to be in a weak magnitude, $(r=0.223 \& \mathrm{p}=$ 0.000 ). There was a strong positive correlation between knowledge about risk one is exposed to incase of nonutilization and the overall knowledge $(r=0.853 \& \mathrm{p}=0.000)$. 


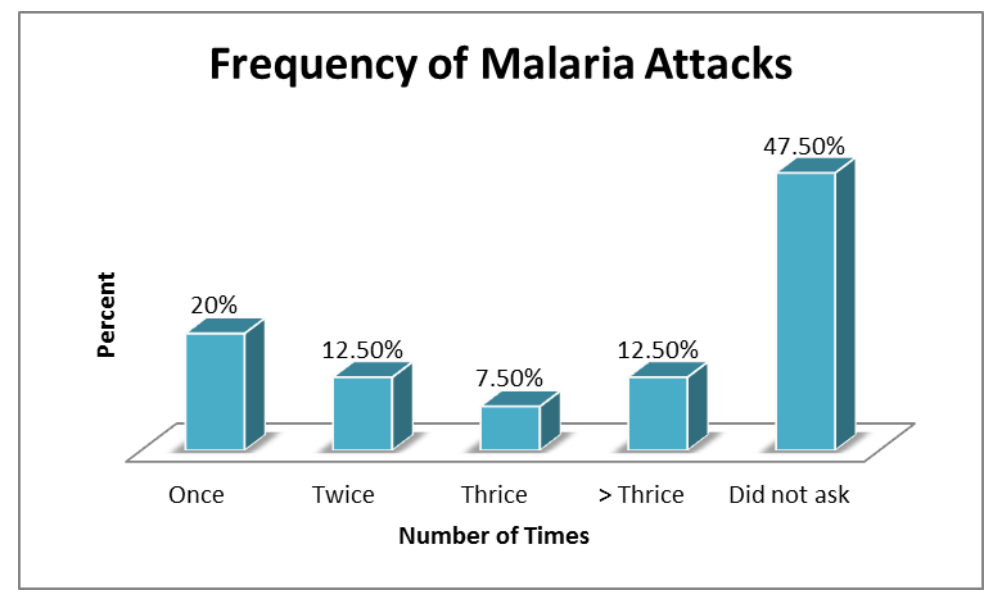

Figure 7. Frequency of malarial attack.

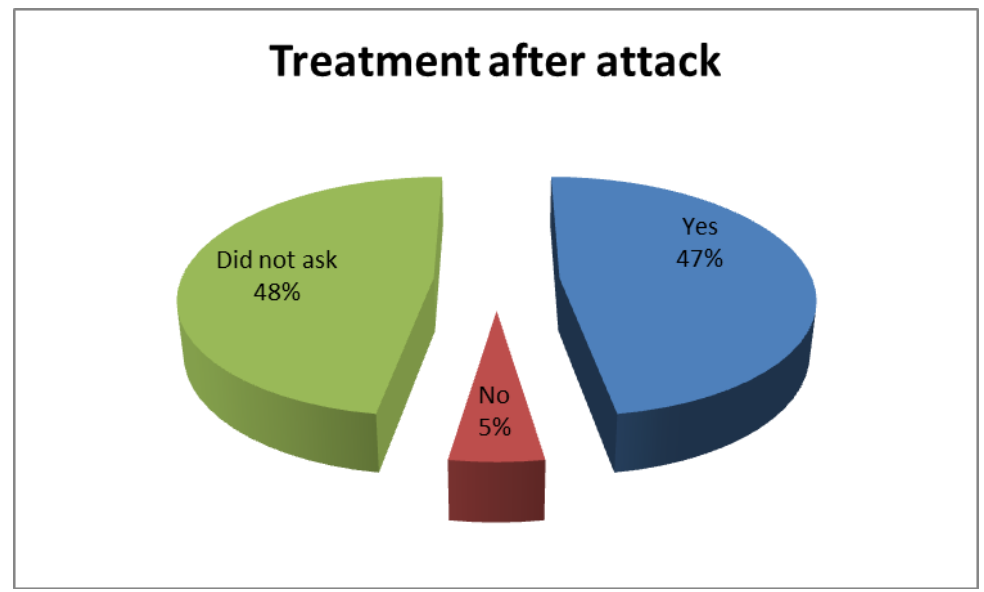

Figure 8. Treatment after malarial attack.

\section{Discussions}

Total of 40 mothers respondents during study were 18 - 41 years with a few (7.5\%) greater than 36 years of age, and most $42.5 \%$ between 18 - 23 years. The study highlights the complex way that women attending the MCH/FP in Webuye District Hospital understand utilization of the ITNs for the prevention of the malaria disease among them and their children who are under five.

The majority of the respondents were mothers that belong to the reproductive age bracket which is between 18 - 35 years of age.

All the respondents had an idea about the ITNs during the time of study, but when asked if they had ITN for personal protection, most of the respondents $90 \%$ possessed while few $10 \%$ said they did not have. This showed awareness of ITNs and confirming accessibility and affordability of major prevention measures for mothers attending $\mathrm{MCH} / \mathrm{FP}$.

Those with ITNs 52\% (19) got sick with malaria while 47.2\% (17) were not. Mothers without ITNs 50\% (2) never got sick. These findings are similar to those of other developing country studies, underscoring the way ITNs utilization can be both effective and infective, at same time may coexist in developing societies. Surveys of pregnant women in eastern India showed that bed nets were widely available and used, although other prevention measures, such as chemoprophylaxis and ITNs, were underused [18].

82.5\% respondents and most used the ITNs for covering them-selves and the child, with few $5 \%$ admitting they use ITNs for restricting chicken and $2.5 \%$ for sieving water from river which was in appropriate. In Abuja declaration, most malaria-endemic countries in sub-Saharan Africa adopted intermittent preventive treatment and insecticide treated nets as intervention for prevention of malaria [10]. Roll Back Malaria Initiative targets set 
for 2010, calling for $80 \%$ coverage of intermittent preventive treatment in pregnancy. This exhibits proper use of ITNs among mothers which contrast with high antenatal-clinic attendance. The few who misused seem not given advice about malaria prevention during antenatal clinic visits. Also given the relatively low levels of education, it was not surprising that respondents used prevention items for sieving river water to make safe for use.

Most respondents 50\% said they used ITNs at night, $27 \%$ used day and night with few $10 \%$ using sometimes which is seasonal and 2.5\% protected themselves and child when they remember. The Roll Back Malaria Initiative guidelines published in 2004 and revised in 2009 recommend proportion of women and children who slept under an insecticide-treated net the previous night as the indicator of insecticides-treated net use. This study concurs with [13]. Insecticide-treated net use during the previous night as reported by women has several limitations. Reported use might not be actual use, or use can be seasonal deepening on the perceived nuisance of mosquitoes, and thus the time of survey can affect the rates obtained.

A number of respondents $75 \%$ demonstrated ITNs importance saying they used to prevent mosquito bites, $17.5 \%$ mentioned they prevented insect nuisance, and a few respondents $7.5 \%$ said it was a requirement for mother and child. This showed respondents had great knowledge of malaria, also appeared to have greater access to bed nets.

All respondents $100 \%$ demonstrated full knowledge of malaria, most respondents $72.5 \%$ linked malaria transmission with a mosquito bite and $10.5 \%$ mentioned it was caused by anopheles mosquito, with $15 \%$ saying cold weather being the caused. $2.5 \%$ mentioned too much sugar cane at which is an incorrect cause of malaria transmission. This high number with knowledge could be due to women receiving focused malaria prevention during antenatal clinic visits.

The $52.5 \%$ admitted they had been attacked by malaria for the past one year during the time study was conducted while almost half $47.5 \%$ said they had not experienced malaria attack for the past one year. Of 52.5\% respondents who were attacked (20\% of them attacked once, and $12.5 \%$ attacked more than thrice) and among those attacked $47.5 \%$ received treatment while few $5 \%$ never sought treatment. This finding suggests that there are missed opportunities when women attended clinics but are not given insecticide-treated nets hence the presence that contracts malaria due to lack of protection from mosquito bite.

The difference was found to be highly statistically significant between the overall knowledge about ITN use and malaria attack and level of education $\left(\chi^{2}=58.7, p=0.000\right)$. The educated population are more exposed to knowledge and this could explain the high percent of those using ITNs to prevent malaria attacks compared to less educated.

The study also indicated positive correlation between overall knowledge and ITN utilization $(r=0.449 \& \mathrm{p}=$ 0.000 ) illustrating the magnitude of education among this population in regards to ITN utilization. Also this finding brings to light that if awareness and health education programs are conducted in an intensive and planned manner, then positive health practices can be achieved.

The same was for the frequency of use but it was found to be in a weak magnitude, $(r=0.223 \& p=0.000$ ). There was a strong positive correlation between knowledge about risk one is exposed to incase of non-utilization and the overall knowledge $(r=0.853 \& \mathrm{p}=0.000)$. The population was highly aware of the dangers one would expect when they failed to use the ITN in accordance to their knowledge and that they received from the Hospital (MCH).

\section{Conclusion}

This report shows that the majority of mothers attending MCH/FP used ITNs and this was attributed to the fact that the MOH provides free ITNs for all these mothers as outlined by the first objective. It also showed that malaria morbidity was influenced by various factors including frequency of ITN use and that most respondents interviewed had contracted malaria once before. Morbidity among mothers with or without ITNs almost equals to those never attacked. This indicates that half the mothers with ITNs never used while those without ITNs that never got attacked could use other preventive methods such as ACT or SP.

\section{Recommendations}

Increase knowledge of effective malaria prevention and treatment methods in communities where misconceptions and use of unproven prevention and treatment methods are common. Improving availability of effective malaria control strategies in areas, by encouraging openness to trying new prevention and treatment approaches, 
including management using Artemisinin-based combination therapies (ACTs) and management of malaria in pregnancy using sulfadoxine-pyrimethamine (SP) for prevention of malaria.

Policy makers should focus on improving linkage for malaria control strategies in this population. Introduce improved laboratory diagnosis at the district hospital.

Further research should be done on ITNs comparing the two groups of mothers, those with and without ITNs. Introduction of cross-cutting strategies including information, education, and communication(IEC) for behavior change, as well as effective monitoring and evaluation of utilization, example is United Against Malaria (UAM) using Africa's obsession with football to convey malaria awareness. Advertise Show malaria prevention messages delivered by Africa's biggest soccer stars.

\section{Acknowledgements}

We thank the staff at Webuye District Hospital, especially the DPHO Mr. Tom Mong'are and his deputy Mr. Mkhuyu, Public Health Officer Mr. Thomas Sikuku who assisted us in data collection.

\section{References}

[1] Ministry of Public Health and Sanitation (MPHS) (2007) Kenya Malaria Indicator Survey. Division of Malaria Control MPHS, Nairobi.

[2] NHSSP (2005) Reversing the Trends: The Second National Health Sector Strategic Plan of Kenya. Nairobi.

[3] KDHS (Kenya Demographic and Health Survey) (2008) Demographic and Health Survey Fact Sheet. Ministry of Planning and Economic Development, Nairobi.

[4] KMIS (2007) Malaria Indicator Survey in Kenya. Ministry of Public Health and Sanitation, Nairobi.

[5] Ministry of Public Health and Sanitation (MPHS) (2009) Kenya Malaria Indicator Survey. Division of Malaria Control MPHS, Nairobi.

[6] Wylie, B.J., Singh, N., Tuchman, J., Sabin, L., Singh, M.P., Brooks, M.I., Yeboah-Antwi, K., Mac Leod, W.B. and Hamer, D.H. (2007) Abstract 535.56th Annual Meeting of the American Society of Tropical Medicine and Hygiene. Philadelphia: Knowledge, Availability, and Utilization of Malaria Prevention Measures during Pregnancy in Jharkland, India.

[7] Gunasekaran, K., Sahu, S.S., Vijayakumar, K.N. and Jambulingam, P. (2009) Acceptability, Willing to Purchase and Use Long Lasting Insecticide Treated Mosquito Nets in Orissa State, India. Acta Tropic, 112, 149-155. http://dx.doi.org/10.1016/j.actatropica.2009.07.013

[8] Oladokun, A., Oladokun, R.E. and Adesina, O.A. (2011) Knowledge and Utilization of Malaria Control Measures by Pregnant and Newly Delivered Mothers in Ibadan, Nigeria. African Health Science, 11, 573-577.

[9] Dellicour, S., Tatem, A.J., Guerra, C.A., Snow, R.W. and ter Kuile, F.O. (2007) Quantifying the Number of Pregnancies at Risk of Malaria in a Demographic Study. PLoS Medicine, 7, e1000221. http://dx.doi.org/10.1371/journal.pmed.1000221

[10] WHO (2004) A Strategic Framework for Malaria Prevention and Control during Pregnancy in the African Region. AFR/MAL/04/01. WHO Regional Office for Africa, Brazzaville.

[11] WHO (2008) Roll Back Malaria Partnership: The Global Malaria Action Plan. World Health Organization, Geneva.

[12] WHO (2009) World Malaria Report, World Health Organization, Geneva.

[13] Baume, C.A., Reithinger, R. and Wodeelhanna, S. (2009) Factors Associated with Use and Non-Use of Mosquito Nets Owned in Oromia and Amhara Regional States, Ethiopia. Malar Journal, 8, 264. http://dx.doi.org/10.1186/1475-2875-8-264

[14] Njagi, J.K. (2002) The Effects of Sulfadoxine/Pyrimethamine Intermittent Treatment and Pyrethroid Impregnated Bed Nets on Malaria Morbidity in Pregnancy and Birth Weight in Bondo District, Kenya. Dissertation, University of Nairobi, Nairobi.

[15] Mabunda, S., Mathe, G., Streat, E., Nery, S. and Kilian, A. (2009) National Malaria Indicator Survey Mozambique (MI-2007). Ministry of Health, National Directorate of Health, Maputo.

[16] (2013) Development Professional Network. www.guardian.com/global

[17] Singh, N., Shukla, N.M. and Sharma, V.P. (1999) Epidemiology of Malaria in Pregnancy in Central India. Bulletin of World Health Organization, 77, 567-572.

[18] Wylie, B.J., Singh, N., Tuchman, J., Sabin, L., Singh, M.P., Brooks, M.I., Yeboah-Antwi, K., Mac Leod, W.B. and Hamer, D.H. (2007) Abstract 535.56th Annual Meeting of the American Society of Tropical Medicine and Hygiene. Philadelphia: Knowledge, Availability, and Utilization of Malaria Prevention Measures during Pregnancy in Jharkland, India. 
Scientific Research Publishing (SCIRP) is one of the largest Open Access journal publishers. It is currently publishing more than 200 open access, online, peer-reviewed journals covering a wide range of academic disciplines. SCIRP serves the worldwide academic communities and contributes to the progress and application of science with its publication.

Other selected journals from SCIRP are listed as below. Submit your manuscript to us via either submit@scirp.org or Online Submission Portal.
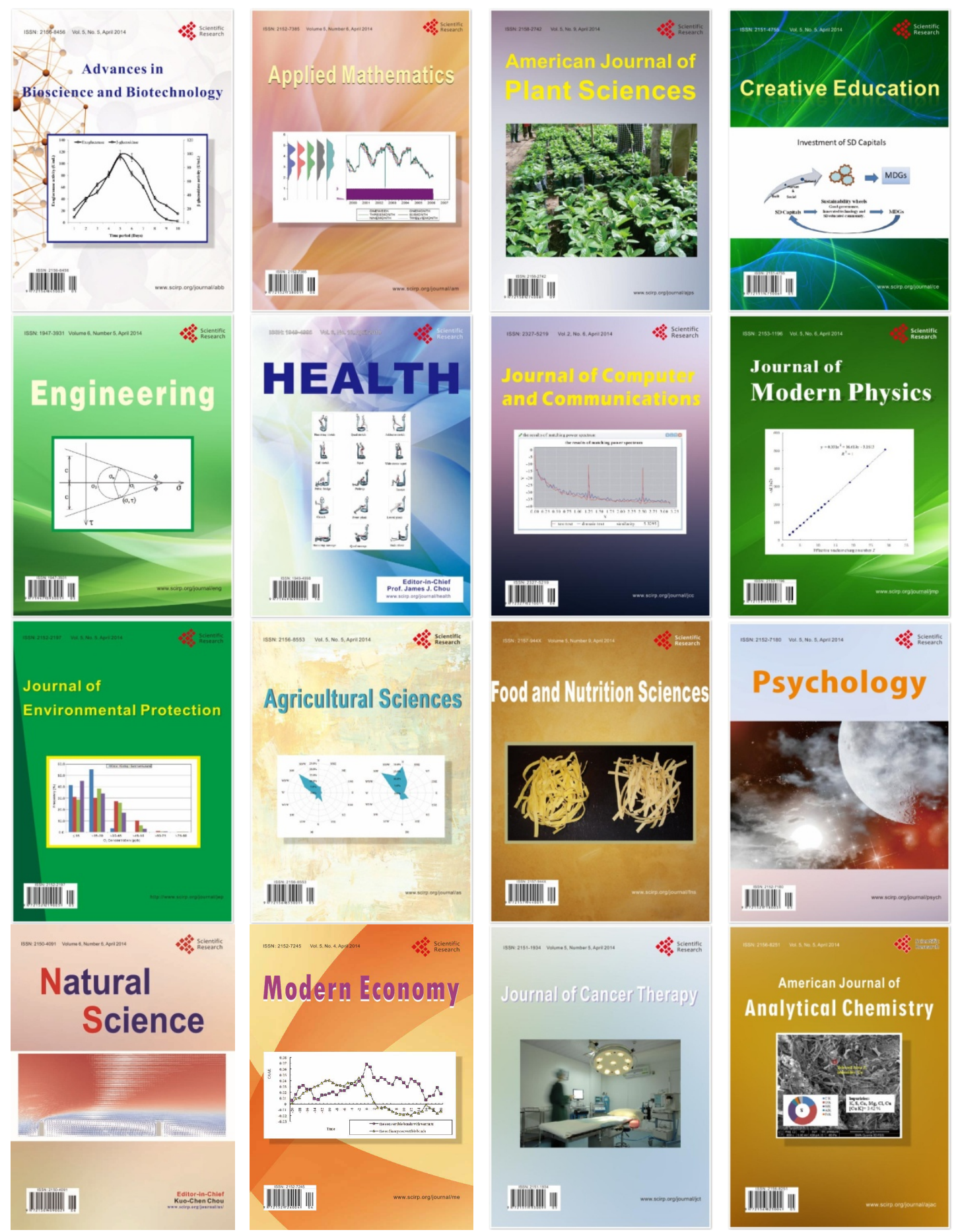